Article

\title{
The Effect of UV Irradiation on the Z-Tetraol Boundary Lubricant
}

\author{
Robert J. Waltman ${ }^{1}{ }^{*}$, John Newman $^{1)}$, Xing-Cai Guo ${ }^{1)}$, John Burns ${ }^{1)}$, Connie Wiita ${ }^{1)}$ and Mina Amo ${ }^{2)}$ \\ ${ }^{1)}$ Hitachi GST \\ 5601 Great Oaks Parkway, San Jose, CA 95119-1003, USA \\ ${ }^{2)}$ Hitachi Ltd. \\ 7-1-1 Omika-cho, Hitachi, Ibaraki 319-1292, Japan \\ *Corresponding author: Robert.Waltman@hitachigst.com
}

( Manuscript received 26 January 2012; accepted 19 March 2012; published 30 May 2012 )

\begin{abstract}
The UV irradiation of Z-Tetraol films on amorphous nitrogenated carbon surfaces was investigated. $\mathrm{COF}_{2}$ evolution, captured in the gas phase, decreased with increasing number of $\mathrm{OH}$ end groups in the order: $\mathrm{Z}>$ Zdol > Z-Tetraol after UV irradiation. Both UV irradiation and annealing on $11 \AA$ Z-Tetraol films produced virtually identical interfacial properties - surface energy as a function of bonded fraction. The UV-irradiated $11 \AA$ Z-Tetraol films also demonstrated equilibrium behavior exhibiting bonding or debonding to the asymptotic $80 \%$ bonded level under ambient conditions. UV-irradiated thick films of Z-Tetraol $(\sim 20-40 \AA)$ exhibited film properties that differed from the non-irradiated films. The oscillations in the polar component of the surface energy, normally observed in Z-Tetraol films and attributed to amphiphilic structuring, disappeared. Terraced flow exhibited a rapidly moving front below $\sim 10 \AA$ compared to the remainder of the irradiated film. Both observations were tentatively attributed to loss of $\mathrm{OH}$ functionality. The latter was verified by chemical extraction and subsequent analyses by NMR and TGA.
\end{abstract}

Keywords: perfluoropolyether, disk lubricant, UV irradiation, surface energy, bonding kinetics

\section{Introduction}

A major challenge facing boundary lubrication in today's hard-disk drives (HDDs) originates from the nanoscale mechanical clearance between the slider and the disk surfaces. While the slider body flies approximately $8-9 \mathrm{~nm}$ above the disk surface, the clearance between the read-write element of the slider and the disk surface is actually as little as several nm due to pole tip protrusion during read/write operations. At these small spacings, the combined effects of the air shear stress, the air bearing pressure, and the van der Waals attractive forces operating between the slider and the disk surfaces can cause lubricant disturbance on the disk surface [1-3]. Isolated and/or periodic lubricant moguls and ripples that form on the disk surface can increase the probability of lubricant transfer to the slider leading to flying instability. Unwanted fly height modulations can hinder read-write functions and increase the probability of intermittent head-disk contacts resulting in a degradation of drive performance. Consequently perfluoropolyether (PFPE) films with increased adhesion to the underlying carbon surface are increasingly being utilized. This is presently achieved in several ways. In the chemical approach, the lubricant itself can be modified to provide a stiffer main chain and/or increase the molecular polarity to provide a boundary lubricant film that is more resistant to the low-flying slider [4-6]. Alternatively, the annealing or the UV irradiation of the boundary lubricant films can increase their adhesion to the underlying carbon surface [7]. UV curing is now widely used in the HDD industry; however, the detailed understanding of the effects of UV irradiation on the boundary lubrication properties of PFPEs is only now becoming better understood [8-11]. The UV irradiation $\left(185,172 \mathrm{~nm}, \mathrm{~N}_{2}\right)$ of the piperonyl-functionalized (AM3001) perfluoropolyether (PFPE) and the non-functionalized Fomblin $\mathrm{Z}$ showed improved corrosion protection which was attributed to increased lubricant bonding and decreased surface energy [12]. The UV irradiation (185 nm, air) of AM3001 decreased both the dispersive and polar surface energies suggesting improved surface coverage [13]. Loss of structure (oscillation) in the polar surface energy as a function of film thickness was attributed to the increased polar interactions between the PFPE and the underlying carbon film. UV irradiation of Z-Tetraol (185 $\mathrm{nm}, \mathrm{N}_{2}$ ) caused increased bonding and photodissociation [14]. Short-term UV irradiation caused a decrease in the polar component of the surface energy, but little or no 
changes in the dispersive surface energy.

In this report, we investigate the effects of UV irradiation $\left(185 \mathrm{~nm}, 172 \mathrm{~nm}, \mathrm{~N}_{2}\right)$ on the thin film properties of the perfluoropolyether Z-Tetraol. We systematically compare as-deposited, annealed and UV-cured Z-Tetraol to quantify possible similarities and differences in the resultant interfacial properties of the boundary lubricant film. Thus lubricant bonding, surface energy, dewetting, and film mobility are directly compared. Thicker films $(50 \AA)$ are employed to extract information on chemical changes induced by the annealing and $\mathrm{UV}$ processes. $\mathrm{COF}_{2}$ yields as a function of the number of $\mathrm{OH}$ end groups provide a systematic approach to obtain further insight into the photochemical quenching of UV-irradiated PFPEs by the hydroxyl end groups. The decreasing $\mathrm{COF}_{2}$ yield with increasing number of $\mathrm{OH}$ end groups indicates a strong preference for UV chemistry to initiate on the hydroxyl end groups. Based on these data we conclude that the UV chemistry of PFPEs may not be ubiquitous but is instead dependent considerably on the end groups accompanying the PFPE main chain.

\section{Experimental}

The substrates used in these studies were $95 \mathrm{~mm}$ diameter AlMg disks of nominally $\leq 2 \AA$ RMS roughness as measured by a Dimension 5000 AFM with a standard AFM tip used in the tapping mode. The typical scan size for these measurements was $5 \mu \mathrm{m} \times 5 \mu \mathrm{m}$ with a scan rate of $0.5 \mathrm{~Hz}$ and 256 lines of resolution. Atop the substrates were sputter-deposited a cobalt-based magnetic recording layer (CoPtCr) and $40 \AA$ of a dual-layered amorphous carbon film comprised of a CVD bottom layer and a sputter-deposited $\mathrm{CNx}$ top layer. The carbon film is comprised nominally of $15 \mathrm{at} \% \mathrm{~N}$. For simplicity, this dual-layered carbon film is referred to as "CNx" throughout this work.

Fomblin Z02, Zdol-2000 and Z-Tetraol-GT 2000 were obtained from Solvay-Solexis. Both Z02 and Zdol-2000 were fractionated by supercritical fluid extraction while Z-Tetraol-GT was used directly as received. The number average molecular weights (Mn) as determined by NMR (nuclear magnetic resonance) were 1650, 1600 and 2120 for Z, Zdol, and Z-Tetraol, respectively. The Z-Tetraol films were applied to carbon surfaces from solvent (HFE-7100) using a standard dip-coating methodology, with a typical concentration of $0.1 \mathrm{~g} /$ liter and a typical disk withdrawal rate of several $\mathrm{mm} / \mathrm{sec}$.

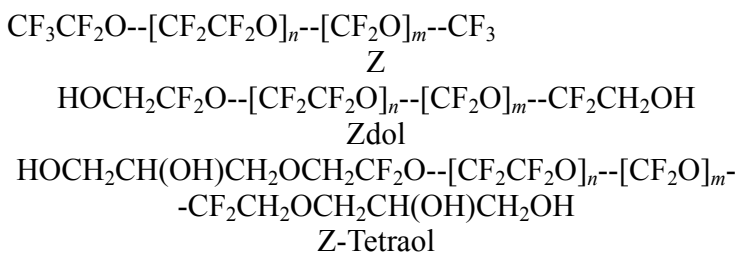

Specular reflection FTIR (Nicolet Magna Model 560) was used to quantify the thickness of the applied perfluoropolyether films. A standard specular reflection adapter (Harrick, New York) was used to measure the PFPE film on the disk. The FTIR absorption band maximum $\left(\sim 1280 \mathrm{~cm}^{-1}\right)$ for each unique PFPE was correlated to film thickness by XPS (Phi Quantum 2000 ESCA System) using a takeoff angle of $45^{\circ}$ and an electron mean free path of $25 \AA$ [15].

The UV irradiation on all Z-Tetraol thin films was accomplished using a Sen Lights (Japan) low pressure $\mathrm{Hg}$ vapor lamp $(185,254 \mathrm{~nm})$ under nitrogen $(\leq 50 \mathrm{ppm}$ $\mathrm{O}_{2}$ ) at nominally $20^{\circ} \mathrm{C}$. The incident power at $185 \mathrm{~nm}$ was $4 \pm 2 \mathrm{~mW} / \mathrm{cm}^{2}$, as measured with a Hamamatsu power meter using a model C8026 controller and a H8025-185 detector (Bridgewater, NJ). The UV irradiation of Z, Zdol and Z-Tetraol liquid films for the gas phase infrared experiments (Figs. 3, 4 only) was accomplished using the Xeradex xenon excimer lamp system (172 nm under nitrogen, $\leq 50 \mathrm{ppm} \mathrm{O}_{2}$ ) from Osram Sylvania Inc., (Danvers, MA). The incident UV power at $172 \mathrm{~nm}$ after transmission through the gas cell quartz window was $2.7 \mathrm{~mW} / \mathrm{cm}^{2}$, as measured with a Hamamatsu Photonics UV power meter using a model C8026 controller and the H8025-172 detector (Bridgewater, NJ).

Throughout the following, we refer to the PFPE films on the disk surface as either being "bonded" or "mobile". To clarify our definition of these terms, we briefly describe the methodology by which these quantities are determined. Following film deposition, the initial film thickness is measured via specular reflectance FTIR. The disks are then washed in 2,3-dihydro-perfluoropentane (Vertrel-XF, DuPont) to remove any soluble PFPE, and the film thickness is remeasured. The quantity retained by the disk is defined to be the amount "bonded", while the portion removed by the solvent wash process is defined as the "mobile" film. For bonded perfluoropolyether, our experiments do not distinguish between physi- and chemi-sorption.

Information on the stability and dewetting of Z-Tetraol films was obtained by directly imaging the disk surface ellipsometrically for lubricant droplets, by ellipsometric imaging of terraced flow, and by contact angle goniometry. For ellipsometric imaging, an optical surface analyzer (OSA) using both s- and p-polarized light was employed [16]. The Q polarized wave (phase contrast signal between the $\mathrm{p}$ - and s-polarized light) provided the highest sensitivity to changes in the lubricant film. Lubricant dewetting was induced by flying a slider over the disk surface as a function of the lubricant film thickness. When the lubricant film thickness exceeded a critical value, i.e., the critical dewetting thickness, lubricant droplets could be observed by OSA.

The energetics of the lubricant/CNx interface was determined as a function of lubricant thickness using contact angle goniometry. Thus, the surface energy for 
the various PFPE films deposited on the $\mathrm{CNx}$ surfaces was determined as a function of lubricant film thickness from contact angle measurements. Hexadecane $\left(\gamma_{l}{ }^{d} \sim \gamma_{l}\right.$ $\left.=27.5 \mathrm{~mJ} / \mathrm{m}^{2}\right)$ and water $\left(\gamma_{l}{ }^{d}=21.8 \mathrm{~mJ} / \mathrm{m}^{2}, \gamma_{l}^{p}=51.0\right.$ $\mathrm{mJ} / \mathrm{m}^{2}$ ) were used as the reference liquids for these measurements. In brief, the contact angle, $\theta$, made between a liquid and a solid surface is related to the free energy of the surface, $\gamma_{s}$, via Young's equation:

$$
\gamma_{l} \cos \theta=\gamma_{s}-\gamma_{s l}
$$

where $\gamma_{l}$ is the surface energy (tension) of the reference liquid, and $\gamma_{s l}$ is the solid-liquid interfacial energy. The dispersive component of the surface energy is determined from contact angle measurements using reference liquids capable of interacting with the surface via dispersive forces only (i.e., hexadecane). In this case, the solid-liquid interfacial energy, $\gamma_{s l}$, is given by [17]:

$$
\gamma_{s l}{ }^{d}=\gamma_{s}^{d}+\gamma_{l}^{d}-2 \sqrt{\gamma_{s}^{d} \gamma_{l}^{d}}
$$

The dispersive component of the surface energy is then obtained by the substitution of eq. (2) into eq. (1),

$$
\gamma_{s}^{d}=\frac{\gamma_{l}^{d}(1+\cos \theta)^{2}}{4}
$$

When the reference liquid is capable of interacting with the surface via both dispersive and polar forces, the interfacial energy can be approximated by [18]:

$$
\gamma_{s l}=\gamma_{s}+\gamma_{l}-2 \sqrt{\gamma_{s}^{d} \gamma_{l}^{d}}-2 \sqrt{{\gamma_{s}^{p}}^{p} \gamma_{l}^{p}}
$$

Substitution into Young's equation readily provides an expression for the polar component of the surface energy. The total surface energy is then the sum of the dispersive and polar components.

The vacuum UV spectra on neat $100 \mu \mathrm{m}$ thick PFPE films (Fig. 3) were taken on a Model U-7000 Vacuum UV (140-300 nm) Spectrophotometer (Hitachi High Technologies Corp.). The PFPE was placed in a liquid cell of $\mathrm{MgF}_{2}$ windows separated by a $100 \mu \mathrm{m}$ thick spacer.

The NMR spectra were obtained on a Bruker Avance $500 \mathrm{MHz}$ spectrometer equipped with a $1 \mathrm{H} / 19 \mathrm{~F}$ cryoprobe. Disk extracts were obtained via one minute, immersion extraction with Vertrel-XF solvent. The extracts from multiple disks were consolidated into a single sample, concentrated into $40 \mu \mathrm{L}$ of a $5: 1$ mixture of perfluorobenzene and d4-methanol and introduced into an NMR capillary tube.

The TGA data was obtained on a TA Instruments Q5000 thermogravimetric analyzer. The NMR samples were introduced into platinum TGA pans, the NMR solvent allowed to evaporate under nitrogen gas flow (40 $\mathrm{mL} / \mathrm{min}$ ) leaving the mobile lube extract. The TGA data was obtained under $\mathrm{N}_{2}$ gas flow $(40 \mathrm{~mL} / \mathrm{min}$ ) in "HiRes" mode.

\section{Results and discussion}

3.1. UV irradiation of thin Z-Tetraol films

The effect of UV irradiation and of annealing on the bonding of $11 \AA$ Z-Tetraol films to the underlying carbon film is first compared. For annealing, we chose $90^{\circ} \mathrm{C}$ to provide relatively efficient bonding kinetics and simultaneously minimize any film thickness loss due to evaporation. As shown in Fig. 1, both processes increase the bonding of Z-Tetraol to the underlying carbon film, resulting in an asymptotic bonded fraction of $\geq \sim 0.9$, considerably greater than can be attained on the basis of diffusive self-stirring under ambient conditions [19]. As expected, the bonding rate is significantly larger for the UV process compared to annealing. Fig. 1 indicates increasing film thickness loss with increasing UV exposure time. This may originate from loss of molecular polarity and/or disk heating with longer UV exposures. The loss of molecular polarity is discussed in Section 3.5. A few tenths of an Angstrom loss in film thickness on the annealed Z-Tetraol (Fig. 1) is also indicated. This may be consistent with very limited evaporative loss expected at this temperature (see Fig. 14 ) or possibly within the expected $\pm 2 \%$ measurement error in FTIR.

Surface energy measurements on PFPE films can provide direct information on the interaction forces that are operative at an interface. The dispersive surface energy per unit area, $\gamma_{s}^{d}$, represents the van der Waals
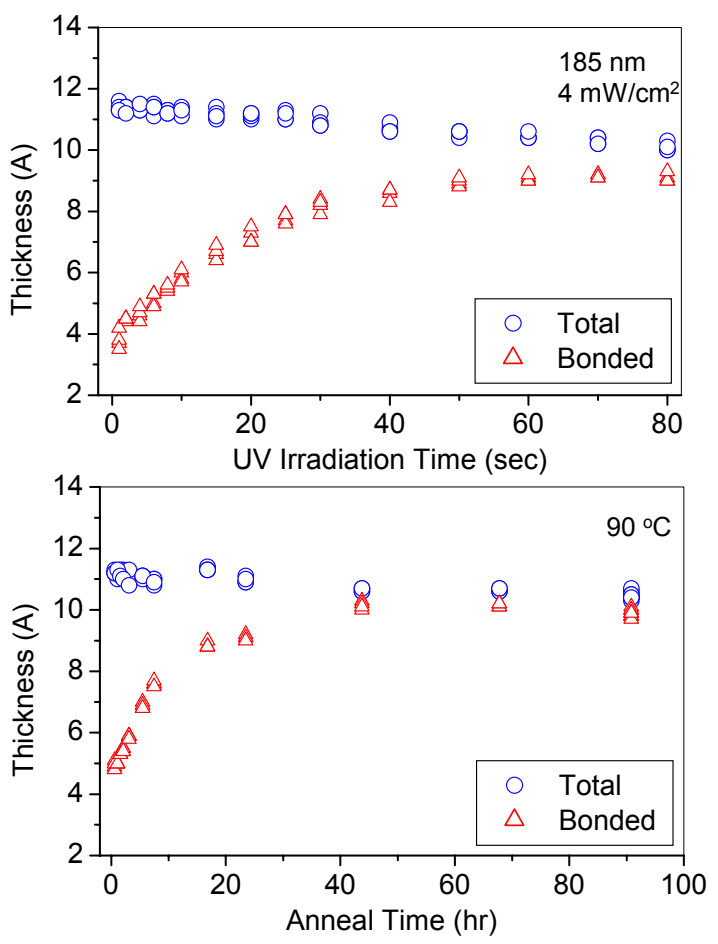

Fig. 1 The (top) UV irradiation and (bottom) annealing of $11 \AA \mathrm{Z}$-Tetraol films on $\mathrm{CNx}$ as a function of time. The UV irradiation used a $185 \mathrm{~nm}$ lamp with an incident power of $4 \mathrm{~mW} / \mathrm{cm}^{2}$. The annealing was conducted at $90^{\circ} \mathrm{C}$ and $1 \%$ relative humidity. 
dispersion forces acting between the perfluorinated ether backbone and the carbon surface while the polar surface energy, $\gamma_{s}{ }^{p}$, represents the polar interactions acting between the PFPE end groups (i.e., hydroxyl) and the polar functional moieties on the carbon surface. The changes in $\gamma_{s}^{d}$ and $\gamma_{s}{ }^{p}$ as a function of bonded fraction for annealed and UV-irradiated Z-Tetraol (11 $\AA$ ) are compared in Fig. 2. The surface energy dependence of both $\gamma_{s}^{d}$ and $\gamma_{s}{ }^{p}$ on the bonded fraction is identical for both UV irradiation and annealing, suggesting both processes act on the PFPE films in a surprisingly similar manner. In both cases, a modest increase in $\gamma_{\mathrm{s}}^{\mathrm{d}}$ is observed as a function of bonded fraction. Based on the data already provided in Fig. 1, we conclude that the increase in $\gamma_{s}^{d}$ as a function of bonded fraction originates from the film thickness loss induced by the UV and annealing processes (see also Fig. 7). This observation cautions against making conclusions on any film thickness-dependent interfacial properties that are measured after UV irradiation based upon as-deposited PFPE film thicknesses.

As regards the polar surface energy, Fig. 2, the decrease in $\gamma_{s}^{p}$ as a function of bonded fraction is
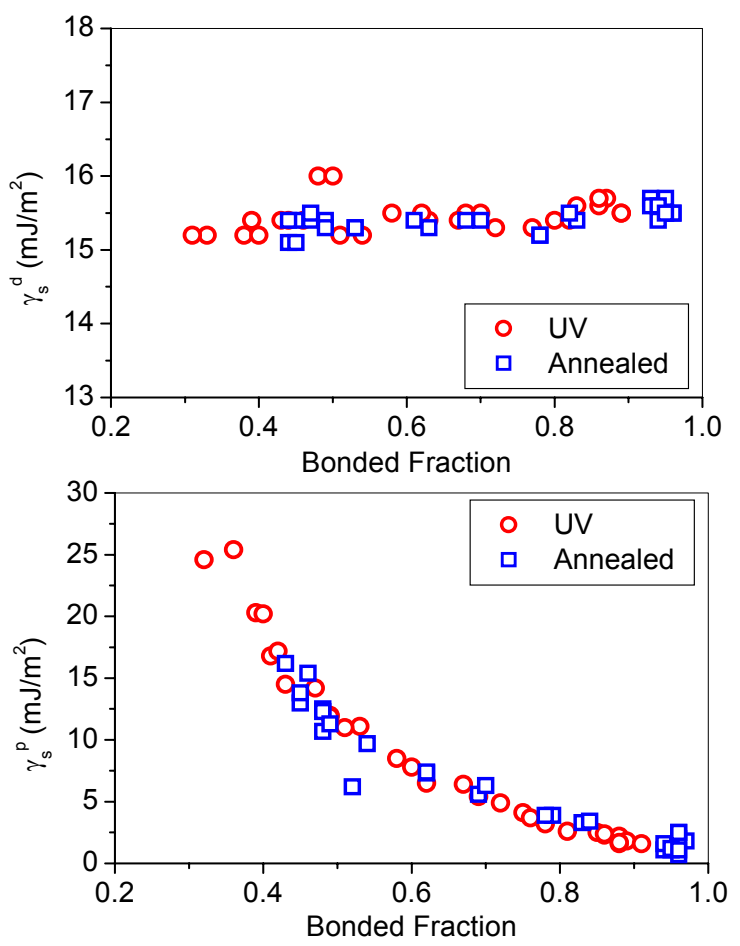

Fig. 2 The changes in the (top) dispersive, $\gamma_{s}^{d}$, and (bottom) polar, $\gamma_{s}^{p}$, surface energies as a function of bonded fraction for $11 \AA$ Z-Tetraol films on CNx. The Z-Tetraol films were separately exposed to UV irradiation $\left(185 \mathrm{~nm}, 4 \mathrm{~mW} / \mathrm{cm}^{2}\right)$ and to annealing $\left(90^{\circ} \mathrm{C}\right)$. The Z-Tetraol film thicknesses were quantified after the UV and annealing treatments.

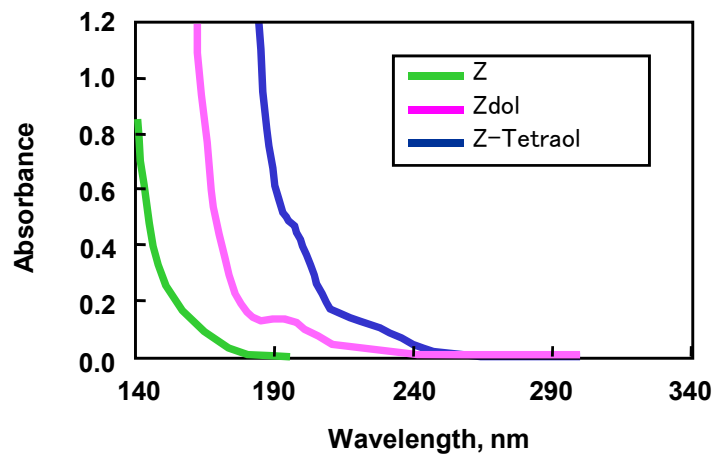

Fig. 3 The vacuum UV spectra for $100 \mu \mathrm{m}$ PFPE films of Z, Zdol and Z-Tetraol.

attributed to the bonding of Z-Tetraol to the underlying carbon film [3]. The hydroxyl end groups of Z-Tetraol interact with the polar sites on the carbon surface resulting in a decrease in $\gamma_{s}{ }^{p}$. Since both sets of data fall on an identical curve, UV irradiation and annealing both act directly on the tetraol adduct. This is corroborated by the observations that UV-irradiated Fomblin $\mathrm{Z}$ bonds to the underlying carbon film [9] while annealed Fomblin $\mathrm{Z}$ does not [20]. Therefore no measurable changes in $\gamma_{s}{ }^{p}$ should be expected in the absence of hydroxyl end groups.

The preferential absorption of UV light by the tetraol adduct is further illustrated in Fig. 3 comparing the vacuum UV spectra of Fomblin Z, Zdol and Z-Tetraol. These data show increasing UV light absorption near $185 \mathrm{~nm}$ with increasing number of hydroxyl end groups. Therefore it is reasonable to assume that the UV chemistry could become increasingly isolated on the tetraol adduct and as a consequence both UV and annealing produce similar thin films while leaving the main chain intact.

Several additional experiments were conducted to directly probe the preferential absorption of UV light by the tetraol adduct. Since the UV irradiation of non-functionalized Fomblin $\mathrm{Z}$ produces $\mathrm{COF}_{2}$ [9], its yield should decrease with increasing molecular polarity (OH groups): $\mathrm{Z}>\mathrm{Zdol}>\mathrm{Z}$-Tetraol. Second, the UV irradiation of chemical mixtures of $Z$-Tetraol and $Z$ should produce less $\mathrm{COF}_{2}$ with increasing Z-Tetraol concentration. In both sets of experiments, the PFPEs were irradiated in a gas cell that allowed both UV and IR transmission for irradiation and detection, respectively [9]. A schematic of the gas cell is shown in the inset of Figure 4. Thus a small quantity of neat PFPE, typically $50-100 \mu \mathrm{m}$ thick $(0.2 \mathrm{~g})$, was coated on a quartz window at the bottom of the UV/IR gas cell. Thicker films were used here to minimize possible surface-induced catalytic effects during the UV irradiation. The cell was purged with nitrogen (approx. $50 \mathrm{ppm} \mathrm{O}_{2}$ ) and isolated by gas valves. Transmission IR spectra were taken through the $\mathrm{NaCl}$ windows while the UV irradiation was done through the quartz windows. 
Referring first to Fig. 4, $\mathrm{COF}_{2}$ was readily detected in the gas phase after the UV irradiation of non-functionalized $\mathrm{Z}$. The $\mathrm{COF}_{2}$ originates from main chain scission [9]. The yield of $\mathrm{COF}_{2}$ clearly decreases with increasing molecular polarity (compare identical 7 min UV irradiation) in the order: $Z>Z$ dol $>$ Z-Tetraol. Even when Z-Tetraol is UV-irradiated for a significantly longer $30 \mathrm{~min}$, no $\mathrm{COF}_{2}$ is detected in the gas phase. Fig. 5 shows the analogous effect of decreased $\mathrm{COF}_{2}$ evolution by the addition of Z-Tetraol into $\mathrm{Z}$ followed by UV-irradiation. These data therefore highlight the complicated nature of the UV photochemistry of PFPEs which can preferentially occur either on the main chain or the end group or both, depending upon the UV or VUV absorption characteristics of the pendant functionality.

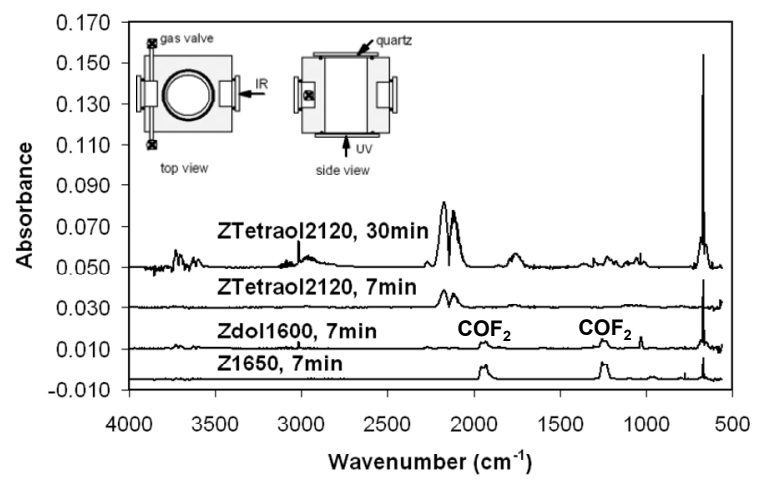

Fig. 4 The gas-phase infrared spectra of Z, Zdol and Z-Tetraol after UV irradiation using a $172 \mathrm{~nm}$ UV lamp at an incident power of 2.7 $\mathrm{mW} / \mathrm{cm}^{2}$. The exposure times are specified in the figure.

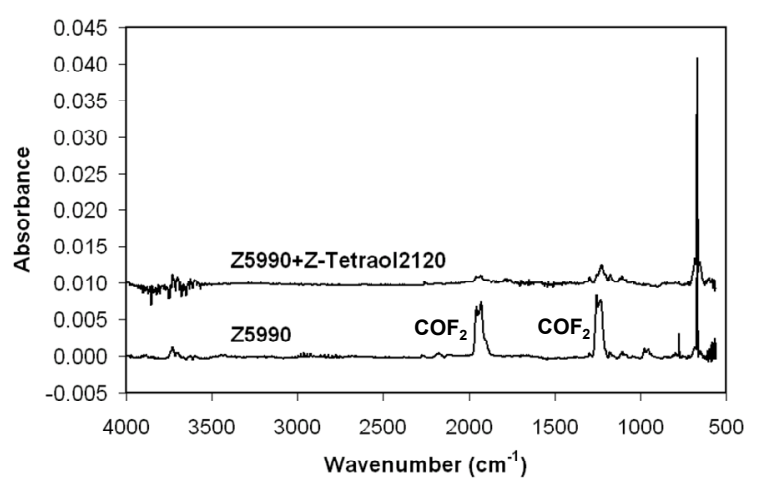

Fig. 5 The gas-phase infrared spectra of $Z$ and a mixture of $Z$ and Z-Tetraol after UV irradiation using a $172 \mathrm{~nm}$ UV lamp at an incident power of $2.7 \mathrm{~mW} / \mathrm{cm}^{2}$ for 7 minutes.
The equilibrium nature of the bonding and surface properties of UV-irradiated Z-Tetraol is next established. Both the changes in the bonded fraction and polar surface energy as a function of time after UV irradiation are shown in Fig. 6 (left) and (right), respectively. Z-Tetraol exhibits the characteristic fractal bonding kinetics reaching an asymptotic bonded fraction of approximately 0.8 under ambient conditions $\left(20^{\circ} \mathrm{C}, 50 \%\right.$ $\mathrm{RH})$ [19]. When the bonding kinetics of UV-irradiated $\mathrm{Z}$-Tetraol is monitored under identical conditions, the bonded fraction converges to the same asymptotic value of $\sim 0.8$ irregardless of the initial UV bonded fraction. The polar surface energy, being kinetically correlated to the PFPE bonding, exhibits a similar result, Fig. 6 (right). Thus, depending upon storage conditions, the bonded fraction of UV-irradiated PFPEs can change with time. Thus the characterization of any interfacial property that depends on the bonded fraction must be carried out at time-of-test for meaningful conclusions to be made. The "debonding" or displacement of some fraction of the bonded lubricant film on carbon surfaces has been attributed to environmental water. In the absence of water, no debonding is observed [21].
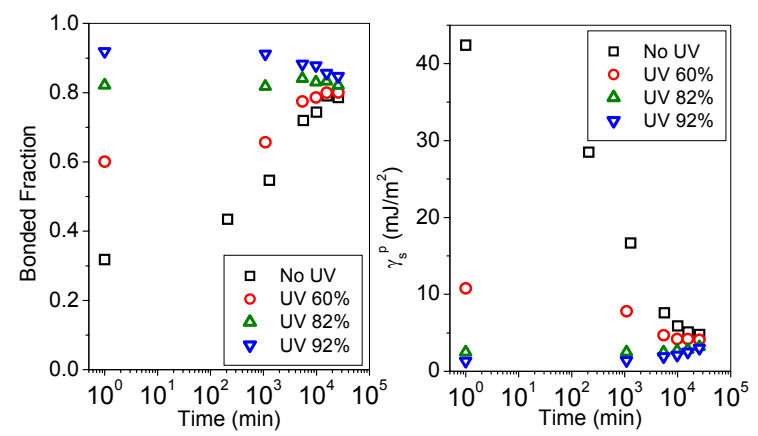

Fig. 6 The ambient (left) bonding kinetics and (right) changes in the polar surface energy of $11 \AA \mathrm{Z}$-Tetraol films on $\mathrm{CNx}$ as a function of time after UV irradiation (185 nm lamp, incident power of $4 \mathrm{~mW} / \mathrm{cm}^{2}$ ).

\subsection{Surface energy versus film thickness}

For PFPE lubricants terminated with functional end-groups, the lubricant film forms a layered structure due to intermolecular interactions between the polar sites on the carbon surface and the PFPE end groups. This surface-induced ordering produces oscillations in the polar component of the surface energy $\left(\gamma_{s}^{p}\right)$ as a function of film thickness. In order to allow for the oscillations in $\gamma_{s}^{p}$ to develop, sufficient lubricant bonding to the underlying carbon film is necessary. Therefore, these measurements were made approximately six weeks after lubricant film application. During this storage time, the disks were sealed in a cassette and stored in partial vacuum (400 mbar) to minimize contamination adsorption from the ambient. 
Fig. 7 shows the dispersive surface energy $\left(\gamma_{s}^{d}\right)$ as a function of film thickness for Z-Tetraol (top) and UV-irradiated Z-Tetraol (bottom) on CNx. The $\gamma_{s}^{d}$ decreases monotonically with increasing film thickness in all cases, approaching what is expected to be the bulk dispersive surface energy. Quantitatively, the dependence of $\gamma_{s}^{d}$ on the applied lubricant film thickness $h$, i.e., $\gamma_{s}^{d}(h)$, can be described using $\gamma_{s}^{d}(h)=\gamma_{l}^{d}+\Delta \gamma^{d}(h)$ where:

$$
2 \Delta \gamma^{d}(h)=\frac{1}{12 \pi} \frac{\mathrm{A}^{*}}{\left(d_{o}+h\right)^{2}}
$$

$d_{o}$ is a constant and $\mathrm{A}^{*}$ is the "effective" Hamaker constant [22]. The $d_{o}$ values used to fit the dispersive surface energy curve via Eq. (5), $d_{o}=2.5 \AA$, is indicative that the interaction between the PFPE main chain and the underlying carbon film is relatively weak and hence cannot provide significant adhesion. The fits to the experimental dispersive surface energy data using these $d_{o}$ values provide an $\mathrm{A}^{*}$ equal to $1.9 \times 10^{-19} \mathrm{~J}$ for
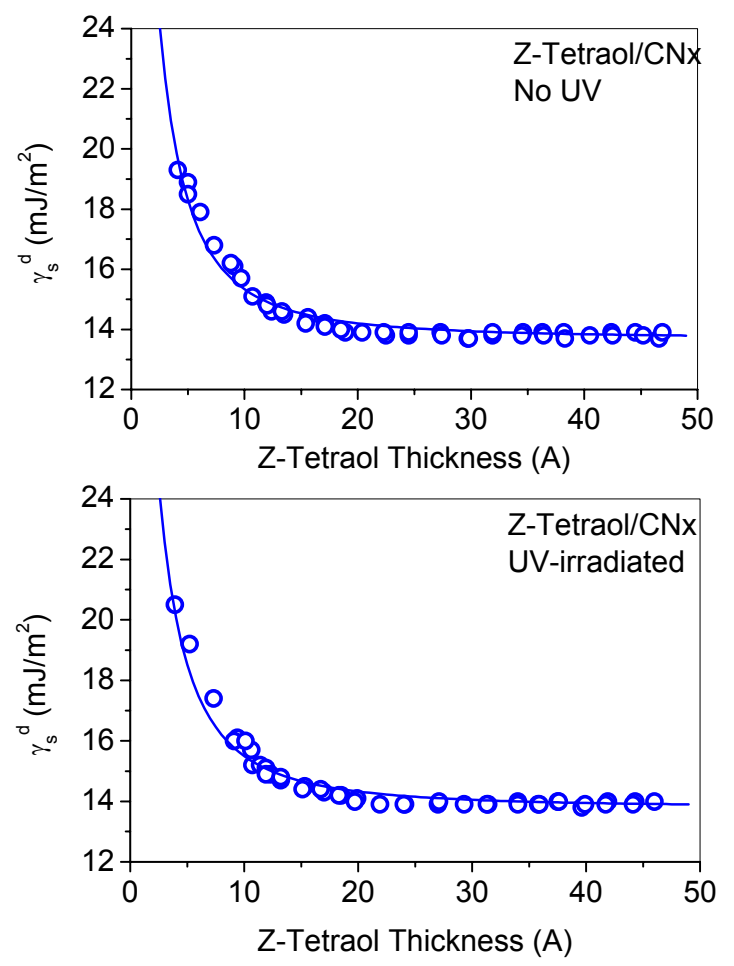

Fig. 7 The changes in the dispersive $\left(\gamma_{s}{ }^{d}\right)$ surface energy as a function of Z-Tetraol film thickness on $\mathrm{CNx}$ without UV irradiation (top) and with UV irradiation (bottom). The UV irradiation was for $20 \mathrm{sec}$ at all film thicknesses using a $185 \mathrm{~nm}$ UV lamp with an incident power of $4 \mathrm{~mW} / \mathrm{cm}^{2}$. The Z-Tetraol film thicknesses were quantified after the UV irradiation. No dewetting was observed by OSA for the films interrogated here.
Z-Tetraol, and $2.0 \times 10^{-19} \mathrm{~J}$ for UV-cured Z-Tetraol, respectively. They are essentially identical; at least, a strong UV effect on the dispersive surface energy is not indicated.

The corresponding polar surface energy as a function of film thickness is shown in Fig. 8. Here significant differences are observed. First, a distinct oscillation in $\gamma_{s}^{p}$ is observed only for Z-Tetraol without the UV irradiation. As shown in the figure, the structure is propagated through several or more monolayers. For UV-irradiated Z-Tetraol, the anticipated oscillation in $\gamma_{s}^{p}$ has virtually vanished. Second, the magnitude of $\gamma_{s}{ }^{p}$ is significantly smaller for the UV-irradiated samples of film thickness greater than approximately $7 \AA$, although this would be expected on the basis of the increased Z-Tetraol bonding (Fig. 6). Below approximately $7 \AA$, the $\gamma_{s}{ }^{p}$ is observed to rapidly increase in both cases due to a lack of lubricant film coverage of the underlying carbon film. For Z-Tetraol, the first minimum in $\gamma_{s}{ }^{p}$
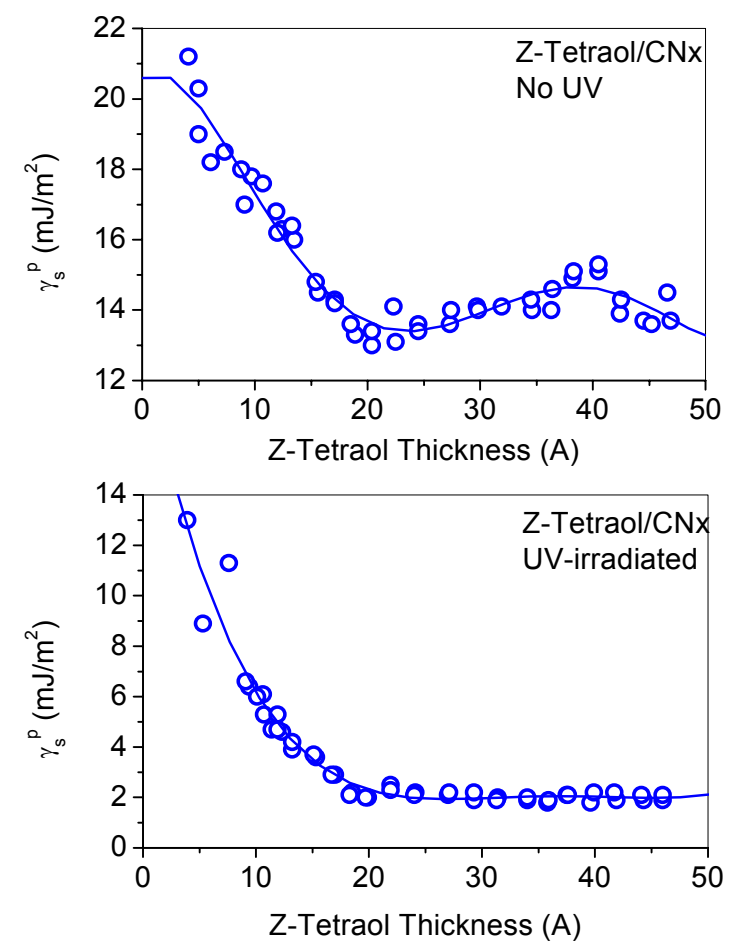

Fig. 8 The changes in the polar $\left(\gamma_{s}^{p}\right)$ surface energy as a function of Z-Tetraol film thickness on $\mathrm{CNx}$ without UV irradiation (top) and with UV irradiation (bottom). The UV irradiation was for $20 \mathrm{sec}$ at all film thicknesses using a $185 \mathrm{~nm}$ UV lamp with an incident power of 4 $\mathrm{mW} / \mathrm{cm}^{2}$. The Z-Tetraol film thicknesses were quantified after the UV irradiation. The solid lines are polynomial fits of the experimental data. No dewetting was observed by OSA for the films interrogated here. 
corresponds to the film thickness at which the surface becomes saturated with lubricant, i.e., the monolayer film thickness, which is approximately $22 \AA$. For the UV-irradiated Z-Tetraol, the minimum in $\gamma_{s}{ }^{p}$ is no longer observed. The removal of the oscillation in $\gamma_{s}^{p}$ may possibly be due to the reduced number of free hydroxyl groups as a result of UV-induced bonding and/or changes in molecular polarity. Fig. 9 compares the bonded thickness of Z-Tetraol as a function of film thickness with and without UV irradiation, taken immediately after the surface energy measurements (Figs. 7, 8) were made. Higher bonding is assumed to be equivalent to a reduced number of $\mathrm{Z}$-Tetraol free hydroxyls. Within the first monolayer $(\sim 22 \AA)$, the data in Fig. 9 indicates that the bonded fraction for the UV-irradiated Z-Tetraol is approximately 0.68 compared to 0.36 for the non-irradiated Z-Tetraol. Consequently amphiphilic layering may be more probable for the non-irradiated Z-Tetraol. For the Zdol system, the magnitude of the oscillations was observed to decrease with increasing molecular weight or equivalently, with decreasing number of hydroxyl groups [20]. Similar observations of loss in film structure in the polar surface energy after UV irradiation have been reported by other workers $[13,14]$.

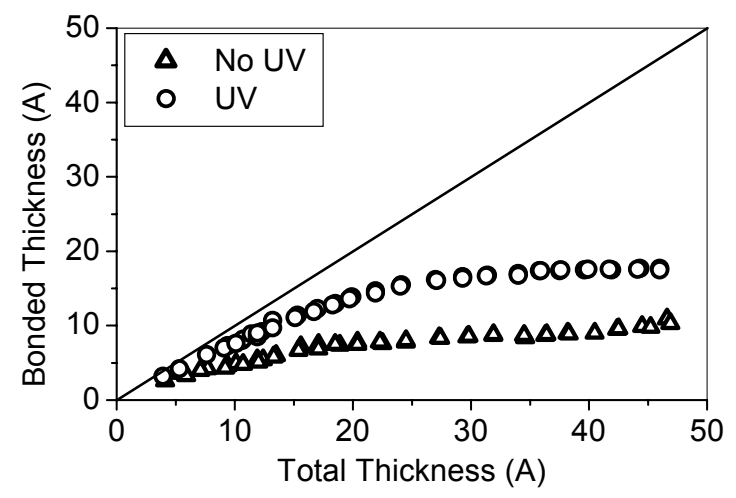

Fig. 9 The bonded thickness for Z-Tetraol as a function of total film thickness on $\mathrm{CNx}$ with and without UV irradiation. The UV irradiation was for $20 \mathrm{sec}$ at all film thicknesses using a $185 \mathrm{~nm}$ UV lamp with an incident power of $4 \mathrm{~mW} / \mathrm{cm}^{2}$.

\subsection{Film stability}

Ellipsometric imaging of UV-irradiated and non-irradiated Z-Tetraol films as a function of $\mathrm{CNx}$ film thickness was used to corroborate the surface energy data for critical dewetting. As shown in Fig. 10, Z-Tetraol films having thicknesses $\leq \sim 22 \AA$ are contiguous and display no signs of dewetting. However, when the Z-Tetraol film thickness is increased to above its monolayer, $23 \pm 1 \AA$, the thermodynamic instability causes the formation of lubricant "droplets" which are readily observed as the darker spots on the imaged surface. UV-irradiated Z-Tetraol films appear to dewet at a slightly thicker 24 to $30 \AA$. Table 1 summarizes the critical thickness for dewetting determined by the various methods discussed thus far.

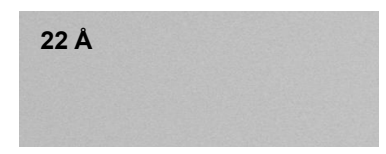

$22 \AA$
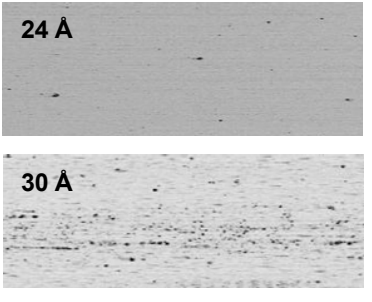

$24 \AA$

$30 \mathrm{~A}$

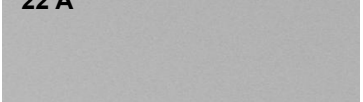

Fig. 10 OSA images identifying the critical thickness for dewetting, $h_{\mathrm{c}}$, for Z-Tetraol on $\mathrm{CNx}$ without UV irradiation (left) and with UV irradiation (right). The UV irradiation was for $20 \mathrm{sec}$ at all film thicknesses using a $185 \mathrm{~nm}$ UV lamp with an incident power of 4 $\mathrm{mW} / \mathrm{cm}^{2}$. The PFPE droplets are identified by the dark spots on the disk surface. The dewetting was induced by flying a head over the disk surfaces as a function of Z-Tetraol film thickness. The films showed no evidence of dewetting prior to the head-flying.

Table 1 Summary of critical dewetting thicknesses $\left(h_{\mathrm{c}}\right)$

\begin{tabular}{|c|c|c|}
\hline Z-Tetraol & $\gamma_{s}{ }^{p}$ & OSA \\
\hline No UV & $21 \AA$ & $22 \leq h_{\mathrm{c}} \leq 24 \AA$ \\
\hline UV Irradiated & N/A & $24 \leq h_{\mathrm{c}} \leq 30 \AA$ \\
\hline
\end{tabular}

\subsection{Terraced flow}

Terraced flow profiles for Z-Tetraol films on $\mathrm{CNx}$ are shown in Fig. 11. The first stable flow region is labeled as the "shoulder" in Fig. 11 (top). The absence of flow into the unstable film thickness regime appears in the spreading profile as the "vertical step" [23]. The shoulder height for both Z-Tetraol and UV-irradiated $Z$-Tetraol is the identical $22 \AA$, suggesting the monolayer and hence the critical dewetting thicknesses are the same. This result apparently contradicts the slightly higher dewetting thickness observed ellipsometrically for UV-irradiated Z-Tetraol in Fig. 10. Since the films used to develop the terraced flow are substantially thicker than those used for the ellipsometric imaging, it is possible that the Z-Tetraol films used in the terraced flow experiments are not significantly different between the UV-irradiated and non-irradiated films. If we use bonded fraction as a 
criterion for the extent of the UV irradiation effect imparted to the two sets of samples, the bonded fraction is approximately 0.40 and 0.25 for the irradiated and non-irradiated samples, respectively. The bonded fractions do not appear to be significantly different. Conversely, close scrutiny of the spreading front clearly shows the UV-irradiated Z-Tetraol has developed additional "structure" that flows at a higher rate near 8-9 $\AA$ film thickness. A similar thinner, rapidly spreading front was also observed in UV-irradiated AM3001 but was not explained [13]. A higher mobility could be associated with chains having lower molecular weight and/or lower polarity as a result of the UV irradiation. We attribute the enhanced flow primarily to the latter which will be discussed below in more detail.
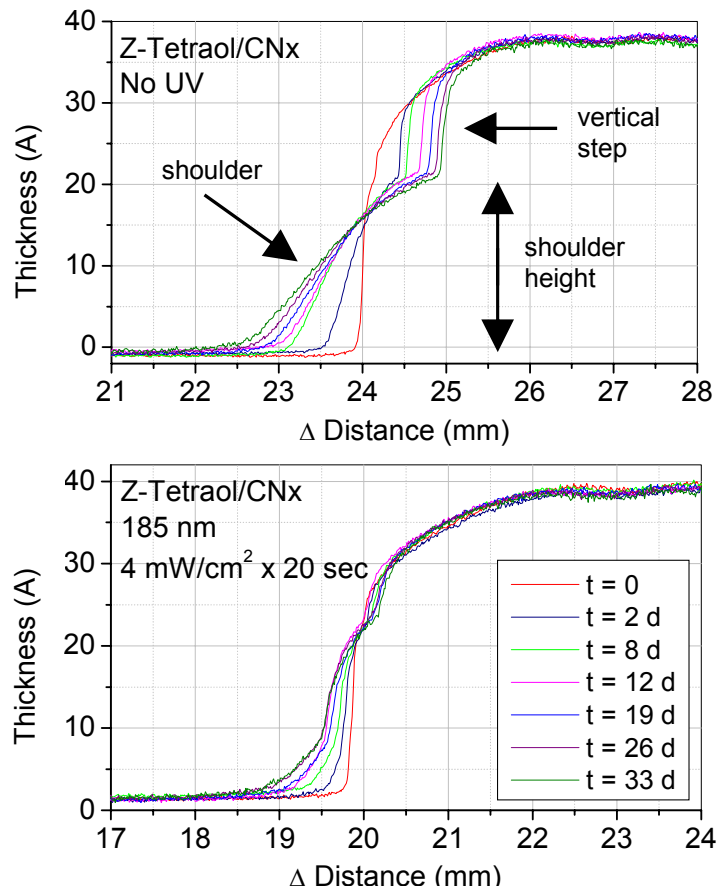

Fig. 11 Terraced flow for Z-Tetraol on $\mathrm{CNx}$ as a function of time without UV irradiation (top) and with UV irradiation (bottom). The UV irradiation was for $20 \mathrm{sec}$ at all film thicknesses using a $185 \mathrm{~nm}$ UV lamp with an incident power of $4 \mathrm{~mW} / \mathrm{cm}^{2}$.

The mobility of UV-irradiated and non-irradiated Z-Tetraol can be described by an "effective" diffusion coefficient that scales with time as [23]:

$$
\mathrm{R}(t)=\left(\mathrm{D}_{\mathrm{eff}} t\right)^{1 / 2}
$$

$\mathrm{R}(t)$ is the distance travelled by the film in time $t$ and $D_{\text {eff }}$ is the effective diffusion constant. $D_{\text {eff }}$ fundamentally differs from the conventional diffusion constant used to describe random motion of a polymer chain in a liquid. Since the flow of molecularly-thin films (typically $\leq \sim 50 \AA$ ) is driven by a pressure gradient, as mentioned above, $\mathrm{D}_{\text {eff }}$ is usually equated to $\mathrm{D}_{\text {eff }}=-m h(\mathrm{~d} \Pi / \mathrm{d} h)$ where $m$ is the mobility and $h$ is the film thickness. $D_{\text {eff }}$ is therefore film thickness-dependent [23]. Since all of the films used here are of comparable film thickness, i.e., $39 \pm 1 \AA$, the results show that the flow rate decreases with UV curing. This is vividly summarized in the summary plot of Fig. 12. Table 2 summarizes the $D_{\text {eff }}$ values obtained from the flow data. We caution that $D_{\text {eff }}$ may be a function of bonded fraction and hence the tabulated values are appropriate for the specified bonded fractions.

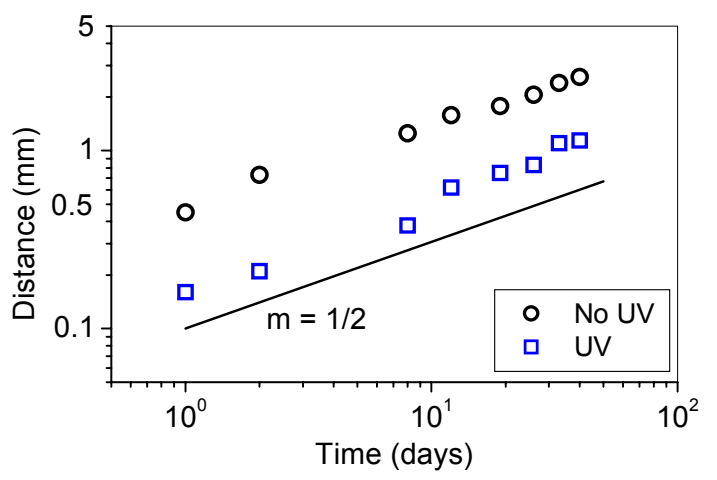

Fig. 12 Terraced flow distance versus time for Z-Tetraol on $\mathrm{CNx}$ with and without UV irradiation.

Table 2 Terraced flow diffusion coefficients

\begin{tabular}{|c|c|c|}
\hline Lubricant & $\begin{array}{c}\text { Z-Tetraol } \\
\text { No UV }\end{array}$ & $\begin{array}{c}\text { Z-Tetraol } \\
\text { UV }\end{array}$ \\
\hline $\mathrm{D}\left(\mathrm{m}^{2} / \mathrm{sec}\right)$ & $1.9 \times 10^{-12}$ & $4.2 \times 10^{-13}$ \\
\hline
\end{tabular}

\subsection{UV irradiation of thicker $50 \AA \mathrm{Z}$-Tetraol films on carbon}

The chemical changes induced by the UV irradiation of Z-Tetraol films were probed using thicker $50 \AA$ films to facilitate characterization. Since NMR is used to quantify the changes in the irradiated films, this methodology depends upon solvent extraction of the irradiated film followed by analysis of the extracted material. Table 3 summarizes the UV irradiation conditions and the measurable changes induced on the disk surface. With increasing UV exposure, the bonded fraction increases substantially. This means the solvent-extractable portion for the subsequent NMR analyses also decreases. Since we could not remove the bonded Z-Tetraol from the carbon surface, data interpretation will be limited because we do not know whether or not the analysis on the extractable fraction is representative of the non-extractable fraction. With this cautionary statement, Table 4 summarizes the chemical 
changes in the extractable fraction of Z-Tetraol as a function of UV exposure. Surprisingly the NMR data indicates that the Z-Tetraol main chain remains largely unaffected by the UV irradiation. In retrospect, this may be the expected result on the basis of the gas phase IR data that indicated decreased $\mathrm{COF}_{2}$ evolution (main chain) with increasing $\mathrm{OH}$ functionality. On the other hand, NMR reveals that there is significant chemical conversion of the tetraol adduct to the dol adduct indicating loss of molecular polarity. This finding is consistent with the disappearance of the expected oscillation in $\gamma_{s}^{p}$ (Fig. 8) and the more rapidly spreading front in UV-irradiated Z-Tetraol in the $\sim 9 \AA$ film thickness range (Fig. 11). The loss in molecular polarity of Z-Tetraol after UV irradiation is shown in the corresponding ${ }^{19} \mathrm{~F}$ NMR spectra of the extracted material, Fig. 13. The end group peaks assigned to the tetraol adduct, -78 and $-80 \mathrm{ppm}$, decrease significantly concomitant with the evolution of the dol adduct, near -81 and $-83 \mathrm{ppm}$, as a function of UV irradiation. For comparison, NMR analysis on annealed Z-Tetraol is shown in Table 5. Similar to UV-irradiated Z-Tetraol, there is loss of the secondary alcohol and an increase in the dol adduct with both disk aging under ambient storage conditions and annealing. In both cases Z-Tetraol bonding also increases with time. NMR analyses clearly indicate that UV irradiation and annealing affect the tetraol adduct in a similar manner. The loss of the secondary alcohol may simply be a manifestation of the enhanced bonding exhibited by Z-Tetraol compared to Zdol with the primary alcohol.

Table 3 Effect of UV processing on Z-Tetraol extract from disk

\begin{tabular}{|c|c|c|}
\hline $\begin{array}{c}\text { UV Process } \\
\text { Time (min) }\end{array}$ & $\begin{array}{c}\text { Total Thickness }(\AA) \\
\text { After UV }\end{array}$ & $\begin{array}{c}\text { Bonded } \\
\text { Thickness }(\AA) \\
\text { After UV }\end{array}$ \\
\hline 0 & 52 & 4 \\
\hline 1 & 51 & 22 \\
\hline 2 & 50 & 36 \\
\hline
\end{tabular}

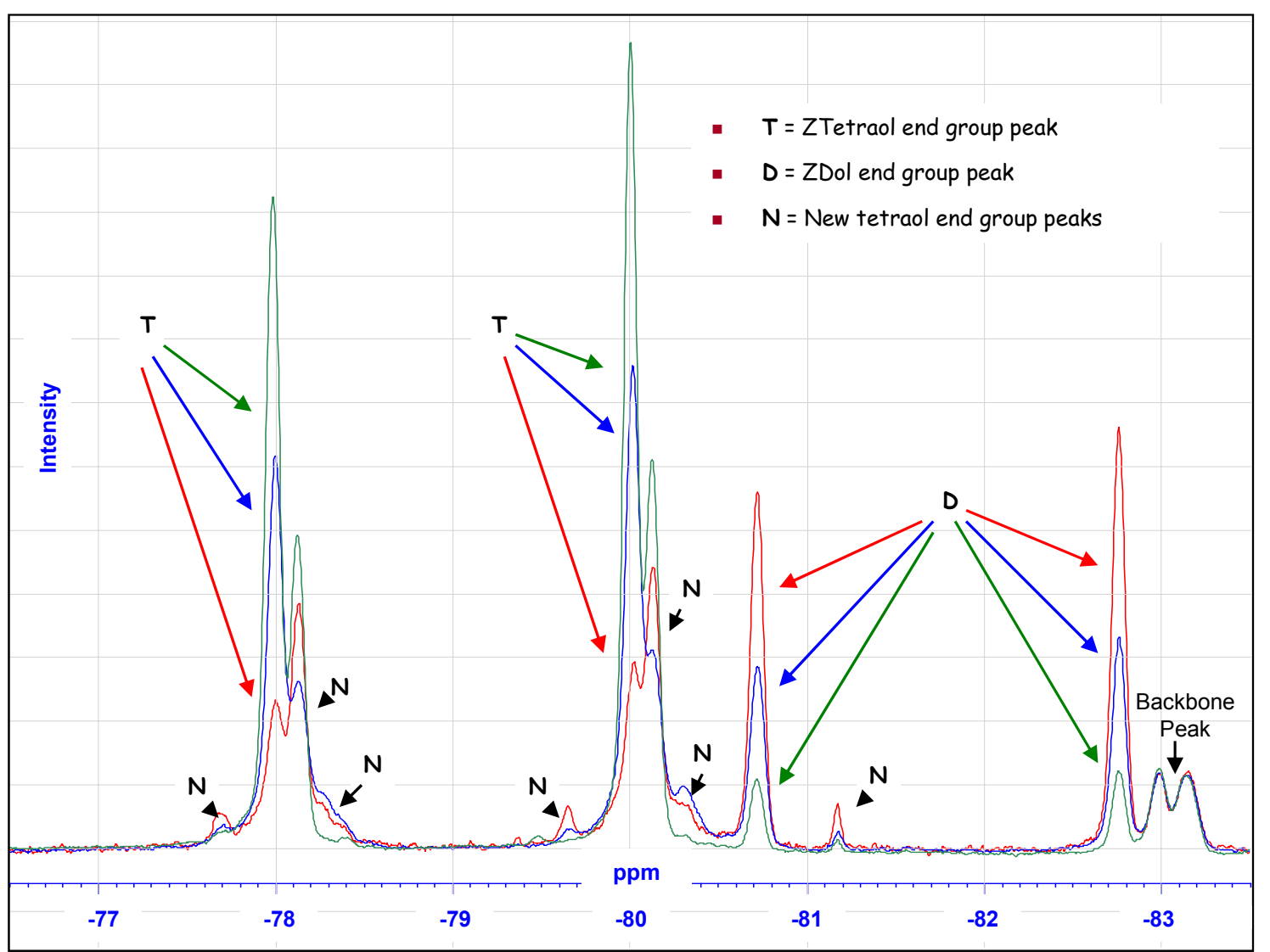

Fig. $13{ }^{19} \mathrm{~F}$ NMR of Z-Tetraol as a function of UV irradiation on solvent-extracted films. The UV irradiation was conducted on $50 \AA$ Z-Tetraol films on CNx for 0 (green), 1 (blue) and 2 (red) minutes using a 185 $\mathrm{nm}$ UV lamp with an incident power of $4 \mathrm{~mW} / \mathrm{cm}^{2}$. 
Table 4 NMR of solvent-extracted Z-Tetraol from disk as a function of UV exposure time

\begin{tabular}{|c|c|c|c|}
\hline $\begin{array}{c}\text { Z-Tetraol Structural } \\
\text { Unit }\end{array}$ & No UV & $\begin{array}{c}1 \mathrm{~min} \\
\mathrm{UV}\end{array}$ & $\begin{array}{c}2 \mathrm{~min} \\
\mathrm{UV}\end{array}$ \\
\hline$-\left(\mathrm{CF}_{2} \mathrm{CF}_{2} \mathrm{O}\right)_{n^{-}}$ & 12.7 & 12.5 & 13.4 \\
\hline$-\left(\mathrm{CF}_{2} \mathrm{O}\right)_{n^{-}}$ & 11.1 & 11.1 & 11.8 \\
\hline $\begin{array}{c}-\mathrm{CF}_{2} \mathrm{CH}_{2} \mathrm{OCH}_{2} \mathrm{CH}(\mathrm{OH}) \\
\mathrm{CH}_{2} \mathrm{OH}\end{array}$ & 1.26 & 0.93 & 0.42 \\
\hline$-\mathrm{CF}_{2} \mathrm{CH}_{2} \mathrm{O}-\mathrm{Rx}$ & 0.64 & 0.69 & 0.83 \\
\hline$-\mathrm{CH}_{2} \mathrm{OH}$ & 0.10 & 0.39 & 0.76 \\
\hline $\mathrm{Mn}$ & 2400 & 2370 & 2470 \\
\hline
\end{tabular}

Table 5 NMR of solvent-extracted Z-Tetraol from disk as a function of ambient aging and annealing at $60^{\circ} \mathrm{C}$

\begin{tabular}{|c|c|c|c|}
\hline $\begin{array}{c}\mathrm{Z}-\text { Tetraol } \\
\text { Structural Unit }\end{array}$ & $\begin{array}{c}\text { No anneal } \\
\text { 1 day aged }\end{array}$ & $\begin{array}{c}\text { No anneal } \\
25 \text { days } \\
\text { aged }\end{array}$ & $\begin{array}{c}\text { Annealed } \\
60^{\circ} \mathrm{C}\end{array}$ \\
\hline$-\left(\mathrm{CF}_{2} \mathrm{CF}_{2} \mathrm{O}\right)_{n^{-}}$ & 9.5 & 9.7 & 9.2 \\
\hline$-\left(\mathrm{CF}_{2} \mathrm{O}\right)_{n^{-}}$ & 9.8 & 10.1 & 8.6 \\
\hline $\begin{array}{c}-\mathrm{CF}_{2} \mathrm{CH}_{2} \mathrm{OCH}_{2} \\
\mathrm{CH}(\mathrm{OH}) \mathrm{CH}_{2} \mathrm{O} \\
\mathrm{H}\end{array}$ & 1.07 & 0.28 & 0.30 \\
\hline$-\mathrm{CF}_{2} \mathrm{CH}_{2} \mathrm{O}-\mathrm{Rx}$ & 0.89 & 1.61 & 1.00 \\
\hline$-\mathrm{CH}_{2} \mathrm{OH}$ & 0.05 & 0.10 & 0.70 \\
\hline $\mathrm{Mn}$ & 2020 & 2040 & 1820 \\
\hline
\end{tabular}

To provide further insight into the chemical changes of the tetraol adduct, the extracted lubricant from the carbon surface were also thermogravimetrically analyzed (TGA), Fig. 14. Fig. 14 (top) shows the weight loss of material as a function of temperature. With increasing UV irradiation, the weight loss onset occurs at lower temperatures. Since the NMR data discussed above quantifies no significant main chain degradation, this observation is consistent with the loss primarily of intermolecular hydrogen bonding due to the conversion of the tetraol adduct to the dol adduct (as opposed to, say, main chain scission). The derivative spectra (Fig. 14 bottom) reveal a substantial broadening of the Z-Tetraol peak in the $\sim 200^{\circ} \mathrm{C}$ region as a function of UV irradiation. The evolution of a small peak in the $300-400^{\circ} \mathrm{C}$ region suggests that perhaps some higher molecular weight component(s) may have been created. Again, the current set of measurements is not able to distinguish possible chemical changes such as end-to-end links or even crosslinks to have taken place. We additionally limit the interpretation of these data to only the solvent-extractable fraction of the UV-irradiated Z-Tetraol films and caution that they do not necessarily represent or reflect the chemical composition of the non-extractable Z-Tetraol film that remained adhered to the disk surface.

\section{Conclusions}

The UV irradiation of Z-Tetraol films on amorphous
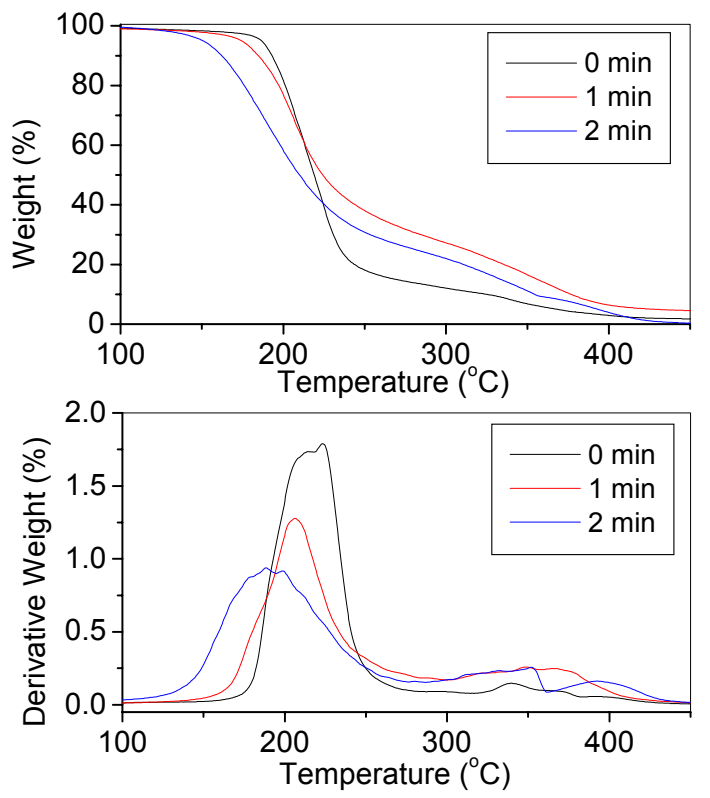

Fig. 14 Thermogravimetric data of Z-Tetraol as a function of UV irradiation on solvent-extracted films. The UV irradiation was conducted on $50 \AA$ Z-Tetraol films on CNx for 0,1 and 2 minutes using a $185 \mathrm{~nm}$ UV lamp with an incident power of 4 $\mathrm{mW} / \mathrm{cm}^{2}$.

nitrogenated carbon films was investigated. $\mathrm{COF}_{2}$ evolution in the gas phase decreased with increasing number of $\mathrm{OH}$ end groups in the order: $\mathrm{Z}>\mathrm{Zdol}>$ Z-Tetraol after UV irradiation. Therefore UV photochemistry was preferentially initiated on the $\mathrm{OH}$ end groups and not the perfluoropolyether main chain. Both UV irradiation and annealing on $11 \AA$ Z-Tetraol films produced a virtually identical surface. Thus the surface energy as a function of bonded fraction was identical. The UV-irradiated $11 \AA$ Z-Tetraol films also demonstrated equilibrium behavior exhibiting bonding or debonding to the asymptotic $80 \%$ bonded level under ambient conditions. UV-irradiated thick films of Z-Tetraol ( 20-40 $\AA$ ) exhibited film properties that differed from the non-irradiated films. The oscillations in the polar component of the surface energy, normally observed in Z-Tetraol films and attributed to amphiphilic structuring, disappeared. Terraced flow exhibited a rapidly moving front below $\sim 10 \AA$ compared to the remainder of the irradiated film. Both observations were tentatively attributed to loss of $\mathrm{OH}$ functionality. The latter was verified by chemical extraction and subsequent analyses by NMR and TGA. For technologically relevant film thicknesses near $10 \AA$, this work demonstrates the high efficiency of the UV process for PFPE bonding compared to annealing and establishes the equivalent interfacial film properties between UV irradiation and annealing. 


\section{References}

[1] Pit, R., Marchon, B., Meeks, S. and Velidandla, V., "Formation of Lubricant "Moguls" at the Head/Disk Interface," Tribol. Lett., 10, 2001, 133-142.

[2] Marchon, B., Karis, T., Dai, Q. and Pit, R., “A Model for Lubricant Flow from Disk to Slider," IEEE Trans. Magn., 39, 2003, 2447-2449.

[3] Waltman, R. J. and Tyndall, G. W., "Lubricant and Overcoat Systems for Rigid Magnetic Recording Media,” J. Magn. Soc. Japan, 26, 2002, 97-108.

[4] Khurshudov, A. and Waltman, R. J., "The Contribution of Thin PFPE Lubricants to Slider-Disk Spacing," Tribol. Lett., 11, 2001, 143-149.

[5] Waltman, R. J., Raman, V. and Burns, J., "The Contribution of Thin PFPE Lubricants to Slider-Disk Spacing. 3. Effect of Main Chain Flexibility," Tribol. Lett., 17, 2004, 239-244.

[6] Guo, X.-C., Knigge, B., Marchon, B., Waltman, R. J., Carter, M. and Burns, J., "Multidentate Functionalized Lubricant for Ultralow Head/Disk Spacing in a Disk Drive," J. Appl. Phys., 100, 2006, 044306:1-8.

[7] Saperstein, D. D. and Lin, L. J., "Improved Surface Adhesion and Coverage of Perfluoropolyether Lubricants Following Far-UV Irradiation," Langmuir, 6, 1990, 1522-1524.

[8] Vurens, G. H., Gudeman, C. S., Lin, L. J. and Foster, J. S., "Mechanism of Ultraviolet and Electron Bonding of Perfluoropolyethers," Langmuir, 8, 1992, 1165-1169.

[9] Guo, X. C. and Waltman, R. J., "Mechanism of Ultraviolet Bonding of Perfluoropolyethers Revisited," Langmuir, 23, 2007, 4293-4295.

[10] Nakakawaji, T., Amo, M., Matsumoto, H., Kato, T. and Iimura, K., "Mechanism of Perfluoropolyether Chains Bonding on Carbon Overcoat Surface by Ultraviolet Irradiation," Tribol. Online, 4, 2009, 88-91.

[11] Jones, P. M., Merzikline, A., Yan, X., Li, L., Dinh, L., Stirniman, M. and Tang, H., "The Influence of Ultraviolet Irradiation on the Surface Chemistry of Ztetraol Magnetic hard Disk: a Combined Temperature Programmed Desorption and X-Ray Photoelectron Spectroscopic Study," Tribol. Lett., 44, 2011, 201-211.

[12] Chiba, H., Takeda, M., Nakamura, N. and Watanabe, K., "Improvements of Lubricant
Performance in Hard-Disk Media by Vacuum Ultraviolet Irradiation," Tribol. International, 36, 2003, 367-369.

[13] Zhang, H., Mitsuya, Y., Imamura, M., Fukuoka, N. and Fukuzawa, K., "Effect of Ultraviolet Irradiation on the Interactions between Perfluoropolyether Lubricant and Magnetic Disk Surfaces," Tribol. Lett., 20, 2005, 191-199.

[14] Chen, H., Chung, P. S. and Jhon, M. S., "Ultraviolet Bonding of PFPE with Carbon Overcoat: Surface Energy and Spreading," IEEE Trans. Magn., 44, 2008, 3702-3705.

[15] Toney, M. F., Mate, C. M. and Pocker, D. J., "Calibrating ESCA and Ellipsometry Measurements of Perfluoropolyether Lubricant Thickness," IEEE Trans. Magn., 34, 1998, 1774-1776.

[16] Cheng, T., Zhao, B., Chao, J., Meeks, S. W. and Velidandea, V., "The Lubricant Migration Rate on the Hard Disk Surface," Tribol. Lett., 9, 2002, 181-185.

[17] Adamson, A. W., "Physical Chemistry of Surfaces," $5^{\text {th }}$ ed., John Wiley and Sons, New York, 1990.

[18] Owens, D. K. and Wendt, R. D., "Estimation of the Surface Free Energy of Polymers," J. Appl. Polym. Sci., 13, 1969, 1741-1747.

[19] Waltman, R. J. and Tyndall, G. W., "Confinement Effects in Monomolecular Perfluoropolyether Films on Amorphous Carbon Surfaces," Thin Solid Films, 517, 2009, 1620-1623.

[20] Waltman, R. J., Tyndall, G. W. and Pacansky, J. "Computer Modeling Study of the Interactions of Zdol with Amorphous Carbon Surfaces," Langmuir, 15, 1999, 6470-6483.

[21] Waltman, R. J., Yen, B. K. and White, R. L., "The Adhesion of Monomolecular Hydroxyl-terminated Perfluoropolyether Liquid Films on the Sputtered Silicon Nitride Surface as a Function of End Group Acidity and Mobility, Tribol. Lett., 20, 2005, 69-81.

[22] Israelachvili, J. N., "Intermolecular and Surface Forces with Applications to Colloidal and Biological Systems," Academic Press, London, 1985.

[23] Tyndall, G. W., Karis, T. E. and Jhon, M. S., "Spreading Profiles of Molecularly Thin Perfluoropolyether Films," Tribol. Trans., 42, 1999, 463-470. 